Vol. 78 - Supl. 2 / 2019

dx.doi.org/10.35366/NTS192E

\title{
Oscilometría de impulso. Recomendaciones y procedimiento
}

\section{Impulse oscillometry system. Recommendations and procedure}

\author{
Laura Gochicoa-Rangel, *,‡ Gabriela Cantú-González, ${ }^{*}$ José Luis Miguel-Reyes, * \\ Luis Rodríguez-Moreno, ${ }^{\ddagger}$ Luis Torre-Bouscoulet*
}

\begin{abstract}
RESUMEN. La oscilometría de impulso (IOS) es una prueba de función respiratoria que permite evaluar la impedancia del sistema respiratorio (Z) a diferentes frecuencias de oscilación. A partir de la Z se derivan la resistencia $(R)$ y la reactancia $(X)$ que son las fuerzas que deben ser vencidas para que el aire pueda ser desplazado dentro y fuera del sistema respiratorio. Mediante los resultados obtenidos de la IOS es posible conocer, de forma indirecta, si el patrón funcional es compatible con obstrucción bronquial o con restricción. La IOS es particularmente útil en aquellos pacientes que presentan síntomas de obstrucción bronquial cuya espirometría en reposo es normal; o bien, para pacientes poco cooperadores en las pruebas dependientes de esfuerzo. Aunque existen recomendaciones internacionales para llevar a cabo la IOS, este documento tiene como propósito orientar al clínico acerca de las ventajas y utilidad de la IOS, así como facilitar su realización mediante la descripción del procedimiento que ha sido estandarizado en el laboratorio de Fisiología Respiratoria del Instituto Nacional de Enfermedades Respiratorias Ismael Cosío Villegas. Finalmente se propone una estrategia simple acerca de la interpretación clínica de la prueba.
\end{abstract}

* Departamento de Fisiología Respiratoria,

Instituto Nacional de Enfermedades Respiratorias Ismael Cosío Villegas, Ciudad de México;

${ }^{\ddagger}$ Centro de Evaluación del Pacientes Asmático Infantil, Centro Médico Santa Teresa. Texcoco, Estado de México. strategy on the clinical interpretation of the test.
Palabras clave: Oscilometría de impulso, procedimiento, función pulmonar, resistencia, impedancia, reactancia, área de reactancia.

\section{Abreviaturas:}

ATS: Sociedad Americana del Tórax.

AX: Área de reactancia.

cm: Centímetros.

Coeh: Coherencia.

DE: Desviación estándar.

E: Elastancia.

EPOC: Enfermedad pulmonar obstructiva crónica.

ERS: Sociedad Europea del Tórax.

VEF $_{1:}$ Volumen espiratorio forzado en el primer segundo.

Correspondencia:

\section{Dr. Luis Torre-Bouscoulet}

Subdirector de Investigación Clínica, Instituto Nacional de

Enfermedades Respiratorias Ismael Cosío Villegas. Calzada de Tlalpan

4502, Colonia Sección XVI, 14080, Ciudad de México.

Correo electrónico: luistorreb@gmail.com

Trabajo recibido: 21-XI-2013; aceptado: 03-XII-2013
ABSTRACT. The Impulse Oscillometry System (IOS) is a lung function test that assesses the respiratory system impedance $(Z)$ at different frequencies of oscillation. From the $Z$ we can get the resistance $Z$ $(R)$ and reactance $(X)$ that are the forces that must be overcome so that the air can be moved in and out of the respiratory system. By the results of the IOS is possible to know, indirectly, if the functional pattern is compatible with bronchial obstruction or restriction. The IOS is particularly useful in those patients with bronchial obstruction symptoms that have a normal spirometry, or, for uncooperative patients in testing effort dependent. Although there are international guidelines for carrying out the IOS, this document is intended to guide the clinician about the advantages and usefulness of the IOS and facilitate their implementation by describing the procedure that has been standardized in our laboratory. Finally, we propose a simple

Keywords: Impulse oscillometry system, procedure, pulmonary function test, resistance, impedance, reactance, area of reactance AX.

Fres: Frecuencia de resonancia.

$\mathrm{Hz}$ : Hertz.

IOS: Sistema de oscilometría de impulso (del inglés impulse oscilometry system). I: Inertancia.

kPa: Kilopascales.

LSN: Límite superior de la normalidad.

PFR: Pruebas de función respiratoria.

R: Resistencia.

X: Reactancia.

Z: Impedancia.

\section{INTRODUCCIÓN}

Las pruebas de función respiratoria (PFR) son de utilidad en el diagnóstico y pronóstico de diversas enfermedades respiratorias. Además, permiten evaluar la respuesta a las intervenciones instituidas y vigilar la evolución funcional de los pacientes. Estas pruebas se pueden clasificar en aquellas 
que evalúan la mecánica de la respiración, el intercambio gaseoso, el control de la respiración y la respuesta integrada al ejercicio. ${ }^{1}$ Las PFR que evalúan la mecánica respiratoria incluyen: la espirometría, la pletismografía, la medición de presiones inspiratoria y espiratoria máximas y las pruebas de reto bronquial. La cooperación del sujeto es central para la buena realización de estas pruebas; es por ello que niños pequeños (usualmente menores de 6 años) o adultos mayores encuentran más dificultad para realizarlas apropiadamente.

En los últimos años, los avances tecnológicos han permitido diseñar equipos que miden parámetros respiratorios sin la cooperación plena del paciente, tales como: el pletismógrafo corporal para infantes, la compresión rápida toracoabdominal, la pletismografía ultrasónica y la medición de la resistencia de las vías aéreas mediante las técnicas de la interrupción y del sistema de oscilometría de impulso (IOS).., Esta última técnica (IOS) tiene la ventaja adicional de que puede dilucidar con razonable precisión el sitio anatómico en donde se encuentra la mayor resistencia al paso del aire. Por otro lado, la medición de la reactancia mediante la IOS, podría ser de utilidad en enfermedades que afectan la distensibilidad toracoabdominal como enfermedades intersticiales, enfermedades neuromusculares o de caja torácica. ${ }^{2}$ La IOS ha sido estandarizada y cada vez es más utilizada, por lo que con el objetivo de continuar mejorando el proceso de calidad en la realización de las PFR, fueron revisadas las recomendaciones internacionales en materia del procedimiento para la realización de la IOS y emitir lineamientos locales que contribuyen a un mejor proceso de estandarización.

\section{Definición}

La IOS es una prueba que evalúa la mecánica respiratoria a través de la aplicación de pequeños pulsos de presión $\left(\sim 1 \mathrm{cmH}_{2} \mathrm{O}\right)$ generados por una bocina, que producen oscilaciones de flujo a una frecuencia determinada que se sobreponen al patrón natural del flujo respiratorio. Los cambios resultantes son captados por un manómetro y un neumotacógrafo permitiendo su análisis subsecuente. ${ }^{3}$ Las frecuencias de oscilación a las cuales se analizan los parámetros funcionales son a 5, 10, 15, 20 y $25 \mathrm{Hertz}(\mathrm{Hz})$.

Los resultados que nos brinda la IOS son:

La impedancia del sistema respiratorio (Z) es la fuerza neta a vencer para mover el gas dentro y fuera del sistema respiratorio. Esta fuerza está compuesta por la resistencia de la vía aérea (R), la elastancia del tórax y pulmón (E) y la inertancia propia del gas y de los tejidos (I). ${ }^{4}$

Los componentes de la $\mathrm{R}$ incluyen la resistencia central (proximal) y periférica (distal) de la vía aérea, como también resistencias del tejido pulmonar y de la pared del tórax.

La reactancia del sistema respiratorio $(X)$ representa el componente reactivo de la impedancia respiratoria e incluyen las fuerzas de masas inerciales del movimiento de la columna de aire en las vías aéreas de conducción, denominada inertancia y propiedades elásticas del pulmón, denominada capacitancia. ${ }^{4}$

La frecuencia de resonancia (Fres) es el punto en el cual, las magnitudes de la reactancia capacitativa e inercial son iguales y tiene un valor igual a cero a distintas frecuencias. ${ }^{5}$ La Fres en niños se registra a frecuencias normalmente más altas que los adultos, cuyo valor normal varía entre 7-12 $\mathrm{Hz}$. La Fres se encuentra siempre aumentada, tanto en trastornos obstructivos como restrictivos. ${ }^{6}$

El área de reactancia $(\mathrm{AX})$ corresponde al índice cuantitativo de la $X$ respiratoria total en todas las frecuencias entre los $5 \mathrm{~Hz}$ y la Fres. Este índice integrativo refleja los grados de cambios de obstrucción en la vía aérea periférica y se correlaciona estrechamente con la frecuencia dependiente de la $R$ y permite evaluar en forma más sensible la respuesta broncodilatadora, así como los cambios que ocurren en tratamientos a largo plazo. ${ }^{7}$

\section{Utilidad}

La IOS es útil en la evaluación de pacientes poco cooperadores en estudios dependientes de esfuerzo pero, además, es particularmente relevante en el estudio de las enfermedades que afectan la vía aérea pequeña, dado que esta región de la anatomía bronquial no es posible evaluarla mediante la espirometría.

\section{Indicaciones}

a. Caracterización de anormalidades funcionales de enfermedades respiratorias crónicas (displasia broncopulmonar, fibrosis quística, asma, enfermedad pulmonar obstructiva crónica [EPOC]). ${ }^{8}$

b. Prueba diagnóstica, particularmente para identificar niños con asma y la respuesta bronquial a un broncodilatador o a un broncoconstrictor. ${ }^{9,10}$

c. Estudiar los mecanismos fisiológicos y la patofisiología de enfermedades que involucran el sistema respiratorio. ${ }^{5,6,11}$

\section{Contraindicaciones}

En términos generales no existen contraindicaciones para la realización del estudio. Es necesario que exista una respiración «tranquila» a volumen corriente; por lo tanto, cualquier circunstancia que afecta el patrón respiratorio de base podría modificar la medición.

\section{Requerimiento del equipo de oscilometría de impulso}

a. El equipo y software debe reunir las recomendaciones 
técnicas internacionales emitidas por la Sociedad Americana del Tórax (ATS) y de la Sociedad Respiratoria Europea (ERS), con lo siguientes requerimientos mínimos: ${ }^{5,12}$

I. Oscilómetro de pulso con neumotacógrafo y con transductor de presión y flujo.

II. Jeringa de $3 \mathrm{~L}$ para la calibración de volumen.

III. Capacidad de medición de volumen de 0.5 a 8.00 $\mathrm{L}$ (con exactitud de $\pm 3 \%$, medido con jeringa de $3.00 \mathrm{~L}$ ), flujos de 0 a $14 \mathrm{~L} / \mathrm{s}$, y tiempo de registro de al menos 30 segundos.

IV. Resistencia de $0.2 \mathrm{kPa}$ para verificación de calibración de presión.

V. Pantalla para presentación de las gráficas de flujo, $R, X$ y coherencia (Coeh).

VI. Impresión del reporte, en el cual debe incluirse la información del paciente, los valores de referencia, los principales valores de oscilometría: $R, X$ y $Z$ a las diferentes frecuencias $(5,10,15,20,25,30 \mathrm{~Hz})$, los gráficos de $\mathrm{R}$ y $\mathrm{X}$ para las tres maniobras; y si la prueba incluye estudio con broncodilatador, incluir los mismos parámetros después de la administración de broncodilatador.

\section{Otro equipamiento y consumibles para la oscilometría de impulso}

a. Computadora e impresora de acuerdo a requerimientos del equipo de oscilometría.

b. Báscula, estadímetro y cinta métrica para medición de extensión de brazos, cuando se requiera.

c. Termómetros ambientales con una precisión de 1 GC.

d. Boquillas desechables con filtro.

e. Pinzas nasales.

f. Aditamentos para control de infecciones:

I. Acceso a lavado de manos y gel-alcohol

II. Filtros en línea desechables con eficiencia de $>99 \%$ para filtración de virus, bacterias y micobacterias; espacio muerto $<100 \mathrm{~mL}$ y resistencia menor de $1.5 \mathrm{cmH}_{2} \mathrm{O}$ a un flujo de $6 \mathrm{~L} / \mathrm{s}$.

III. Guantes desechables.

IV. Cubreboca quirúrgico para protección general y cuando se requiera, cubreboca N95 con fuga menor al $10 \%$ y una eficiencia de filtrado de $>95 \%$ a un flujo de $50 \mathrm{~L} / \mathrm{min}$.

V. Anteojos de protección

g. Consumibles para el broncodilatador:

I. Salbutamol en inhalador de dosis medida (100 $\mu \mathrm{g}$ por atomización)

II. Cámara de reservorio (espaciador) con un volumen recomendado de al menos $300 \mathrm{~mL}$.

\section{Instrucciones del paciente antes de la prueba}

a. Se debe evitar tabaquismo $2 \mathrm{~h}$ antes de la prueba.

b. No se recomienda el uso de prendas restrictivas de tórax o abdomen como chalecos, corsés o ropa muy apretada.

c. Si la prueba incluye evaluación de la respuesta al broncodilatador y el paciente usa medicamentos broncodilatadores, debe suspenderse la última dosis previa a la prueba (un mínimo de 4 horas para broncodilatadores de corta duración y 12 a 24 horas para broncodilatadores de larga duración), previa autorización del médico tratante.

d. Los pacientes deben mantener cualquier otra medicación de base.

e. No se requiere de ayuno para la prueba, pero se recomienda un alimento ligero.

f. Evitar ejercicio intenso antes de la prueba.

g. Es recomendable aplicar un cuestionario breve que incluya:

I. Historia de tabaquismo, activo o pasivo, número. total de años de fumador y promedio diario de cigarros por día.

II. Historia de exposición laboral a humos o polvos, número total de años de exposición y promedio de horas por día.

III. Historia de síntomas respiratorios: disnea, sibilancias, tos y expectoración.

V. Historia de enfermedades o traumas toracopulmonares.

\section{Preparación del equipo antes de la prueba}

a. Todos los componentes (mangueras, sensores, conectores, etc.) deben ser ensamblados de acuerdo a las instrucciones del fabricante.

b. Encender el equipo y dar tiempo suficiente para el calentamiento del mismo, de acuerdo con las instrucciones del fabricante.

c. La oscilometría diagnóstica se debe realizar en ambientes de temperatura dentro de 17 a $40{ }^{\circ} \mathrm{C}$.

d. Los sensores de flujo deben limpiarse siguiendo las recomendaciones del fabricante y estar libres de partículas que obstruyan el sensor.

e. El oscilómetro debe estar codificado a la altitud o presión barométrica, temperatura y humedad relativa promedio del sitio donde se realiza el estudio.

f. El oscilómetro debe estar codificado con los predichos que ajusten a la población a estudiar. 


\section{Calibración o verificación de calibración del oscilómetro}

a. Calibración: procedimiento por el cual se establece una relación entre el volumen o flujo medido por el sensor y el flujo o volumen real del calibrador (jeringa). Esta maniobra se refiere a un ajuste de ganancia eléctrica del dispositivo.

b. Verificación de calibración: concepto diferente al de calibración. Este procedimiento se usa para validar que el oscilómetro se encuentra dentro de los límites de calibración (exactitud), $\pm 0.3 \%$. Si el dispositivo falla, la verificación debe repetirse. En caso de fracaso repetido, el equipo debe enviarse a mantenimiento o revisión.

c. Procedimiento:

I. El oscilómetro debe ser calibrado o verificado en calibración diariamente para volumen, antes de realizar la prueba, al inicio de labores y cada vez que haya duda de su exactitud y para flujo cada semana.

II. Utilizar una jeringa de $3.00 \mathrm{~L}$ con una exactitud de $\pm 0.5 \%$ del volumen absoluto $(15 \mathrm{~mL})$. Una jeringa dañada o golpeada es considerada potencialmente descalibrada y no debe usarse para este procedimiento.

III. Asegurar una conexión firme y hermética entre la jeringa y el oscilómetro, especialmente cuando no se utilice la jeringa original del equipo.

IV. Abrir la rejilla de resistencia de la parte posterior del equipo.

V. La rutina de calibración debe seguir las recomendaciones del fabricante.

VI. La mayoría de los oscilómetros actuales tienen menús especiales en el programa para realizar y almacenar los datos de calibración.

VII.Las mediciones de calibración deben fluctuar entre 2.91 y $3.09 \mathrm{~L}$ (variación de 3\%). Sin embargo, es recomendable que oscilen entre 2.95 y $3.05 \mathrm{~L}$ (variación de $50 \mathrm{~mL}$ ).

VIII.Para la verificación de la calibración de presión se debe cerrar la rejilla posterior (resistor), colocar la resistencia administrada por el fabricante y realizar una oscilometría convencional. El resultado debe cumplir con una resistencia a $5 \mathrm{~Hz}(\mathrm{R} 5 \mathrm{~Hz})$ entre 0.19-0.21 kPa/L/s y una X a $5 \mathrm{~Hz}(X 5 \mathrm{~Hz})$ de cero.

\section{Preparación del paciente para la prueba}

a. El técnico que realiza la prueba recibe y se presenta con el paciente.

b. Se debe revisar la solicitud médica y confirmar el nombre completo y el número de registro. c. El técnico explica al paciente el objetivo de la prueba. La frase más sencilla recomendada es la siguiente:

«La oscilometría de impulso es una prueba que consiste en respirar tranquilamente a través de una boquilla que sirve para conocer las resistencias que puedan existir en sus bronquios y saber si existe o no obstrucción. Si observamos que existe un aumento en las resistencias, procederemos a administrar un medicamento llamado salbutamol, esperaremos unos minutos y volveremos a realizar la prueba para ver si mejora con la administración del medicamento».

d. Si el técnico en función detecta como contraindicación llanto del paciente (en caso de los niños), debe comunicarlo al supervisor médico para que éste evalúe y supervise la ejecución de la misma.

e. Registrar el consumo de tabaco, el ejercicio físico intenso antes de la prueba y el uso de broncodilatadores.

f. La estatura se mide en centímetros $(\mathrm{cm})$ y con el individuo sin zapatos, en posición completamente erecta, talones juntos y mirando al frente. Para la medición de estatura es recomendable el uso de estadímetros de pared.

g. En los pacientes que no puedan mantenerse de pie o sufran de deformidad de caja torácica, se puede usar la extensión de los brazos como una estimación de la estatura. Se solicita al paciente que extienda al máximo los brazos en direcciones opuestas. Se mide la extensión entre el extremo de los dedos medios de cada mano. La estatura se estima para hombres como extensión de brazos en $\mathrm{cm}$ dividido entre 1.03 y para mujeres dividida entre 1.01.

h. El peso se mide en una báscula calibrada y se registra en kilogramos (kg) en unidades cerradas al 0.5 kg más cercano. El peso no es utilizado en la mayoría de las ecuaciones de referencia para calcular valores normales; pero es útil para fines de interpretación.

i. La edad se registra en años cumplidos al día de la prueba.

j. La prueba debe realizarse con el sujeto sentado. Se deben utilizar sillas sin ruedas y con soporte para brazos. Se coloca al sujeto sentado con el tórax y cuello en posición erguida y con ambos pies apoyados sobre el piso. Si se utiliza una posición diferente, ésta debe de registrarse.

\section{Maniobra de oscilometría}

a. Se explica el procedimiento que se va a realizar y los siguientes conceptos (Figura 1):

I. Estará sentado durante la prueba.

II. Se le colocará una pinza en la nariz para evitar que respire por la misma. 
III. Una persona (personal de salud) o el mismo paciente sostendrá sus mejillas.

IV. Se le colocará una boquilla con filtro en la boca, en la cual no debe meter la lengua, ni morderla, debe sellar los labios alrededor de la misma y respirar tranquilamente (Figura 1).

V. Se le demostrará el ruido que realiza el aparato

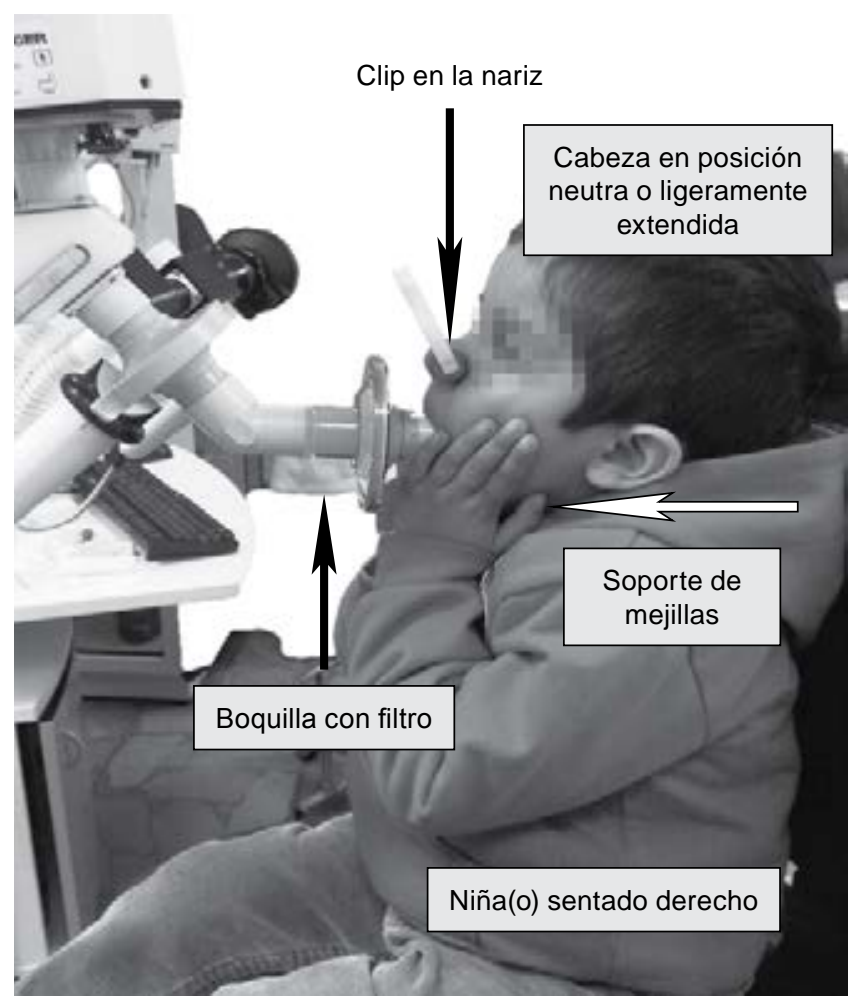

Figura 1: Paciente durante la realización de la IOS. Posición sedente, pinza nasal colocada, sostén en sus mejillas y boquilla con filtro. cada vez que mide la $R$, y se le explicará que no debe de asustarse y que deberá seguir respirando tranquilamente.

b. Una vez explicado el procedimiento se realizan tres mediciones de 30 segundos cada una, las cuales deben tener los siguientes criterios de aceptabilidad:2,13

1. El paciente debe tener al menos cuatro respiraciones en volumen corriente y de forma regular (Figura 2).

2. La morfología de las curvas deben estar libres de artefactos: tos, cierre glótico, respiración agitada (Figuras 3-5).

c. Las mediciones deben de contar con los siguientes criterios de repetibilidad:5,14-16

1. La medición debe durar 30 segundos, si existe algún artefacto, debe eliminarse y recalcularse.

2. Debe de pasar un minuto entre las mediciones realizadas.

3. Debe haber un espectro de frecuencia entre $5 \mathrm{~Hz}$ y $25 \mathrm{~Hz}$.

4. La coherencia que determina la correlación que existe entre las señales que entran y salen debe ser de 0.6 a $5 \mathrm{~Hz}$ y de 0.9 a $10 \mathrm{~Hz}$.

5. Debe existir una variabilidad entre las mediciones menores al $10 \%$ en frecuencias mayores a $5 \mathrm{~Hz}$.

d. Una vez obtenido las primeras tres mediciones se procede a administrar un broncodilatador de acción corta (salbutamol) $400 \mu \mathrm{g}$ en adultos y $200 \mu \mathrm{g}$ en niños, con cámara espaciadora.

e. El paciente permanece en reposo por 20 minutos.

f. Se repite el procedimiento.

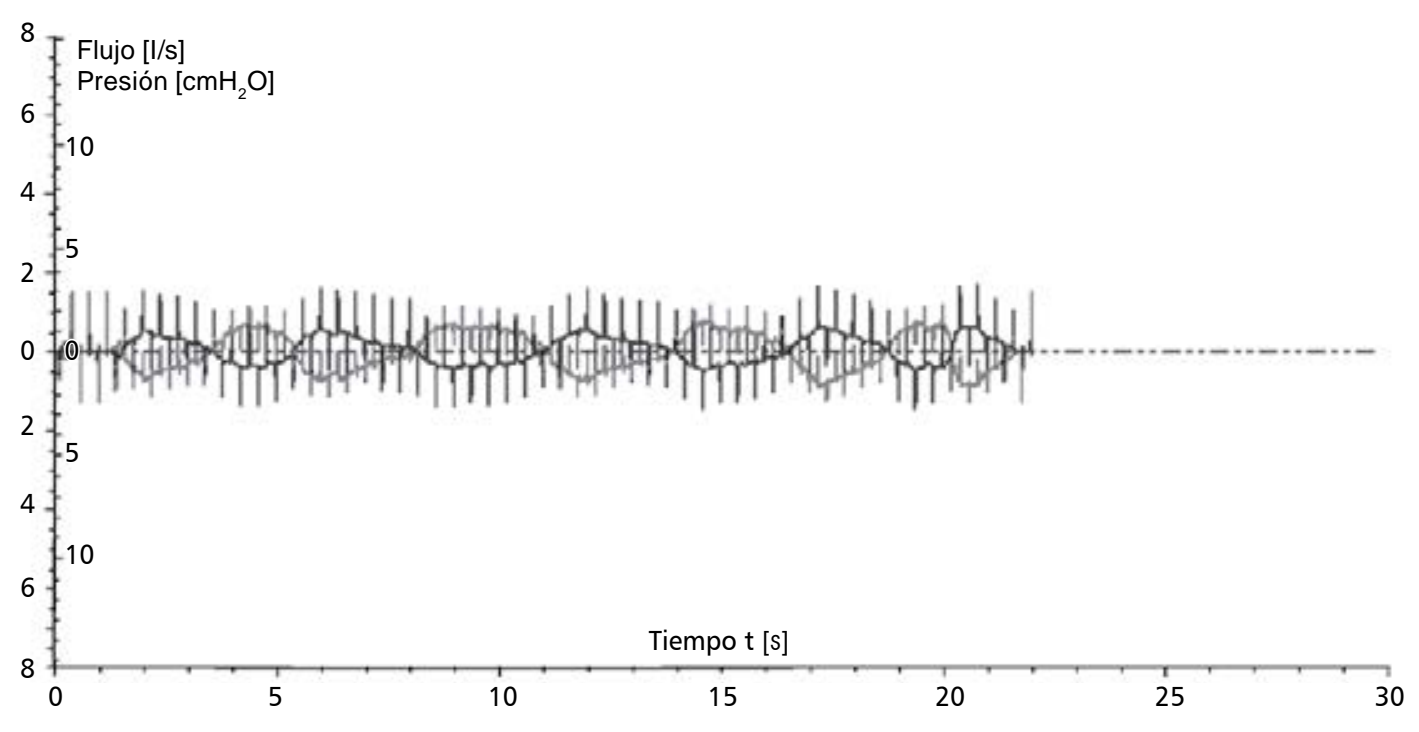

Figura 2:

Gráfico que muestra la respiración regular donde se combina el flujo y la presión. 


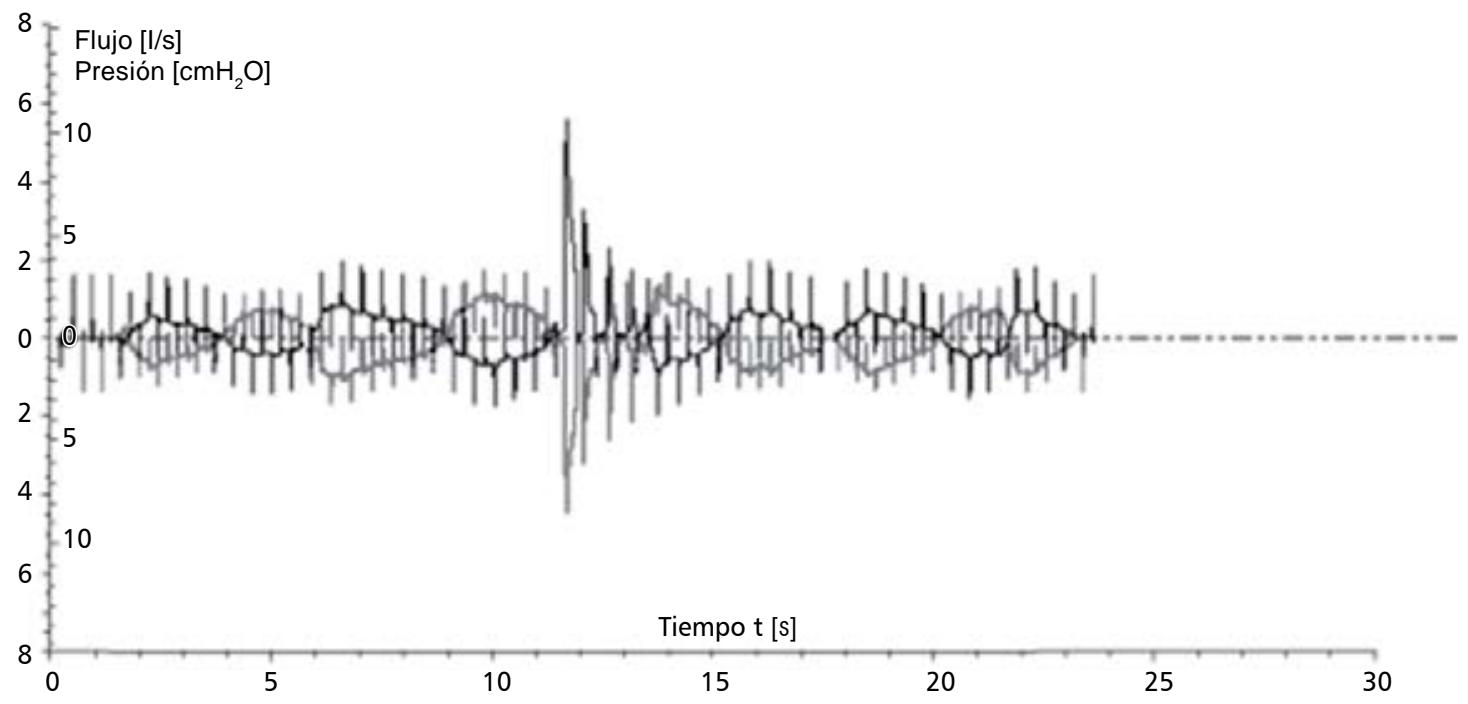

Figura 3:

Artefacto:

tos durante la prueba.

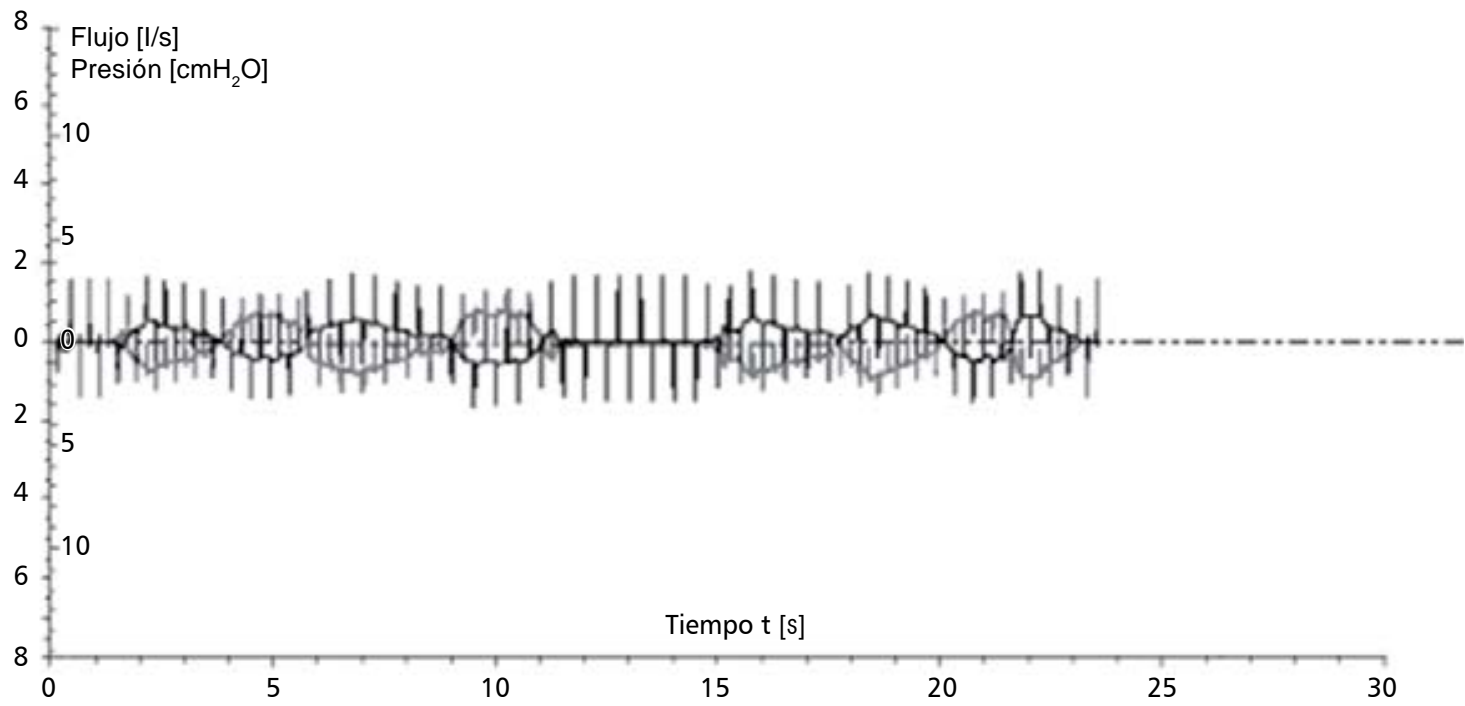

Figura 4:

Artefacto: cierre glótico.

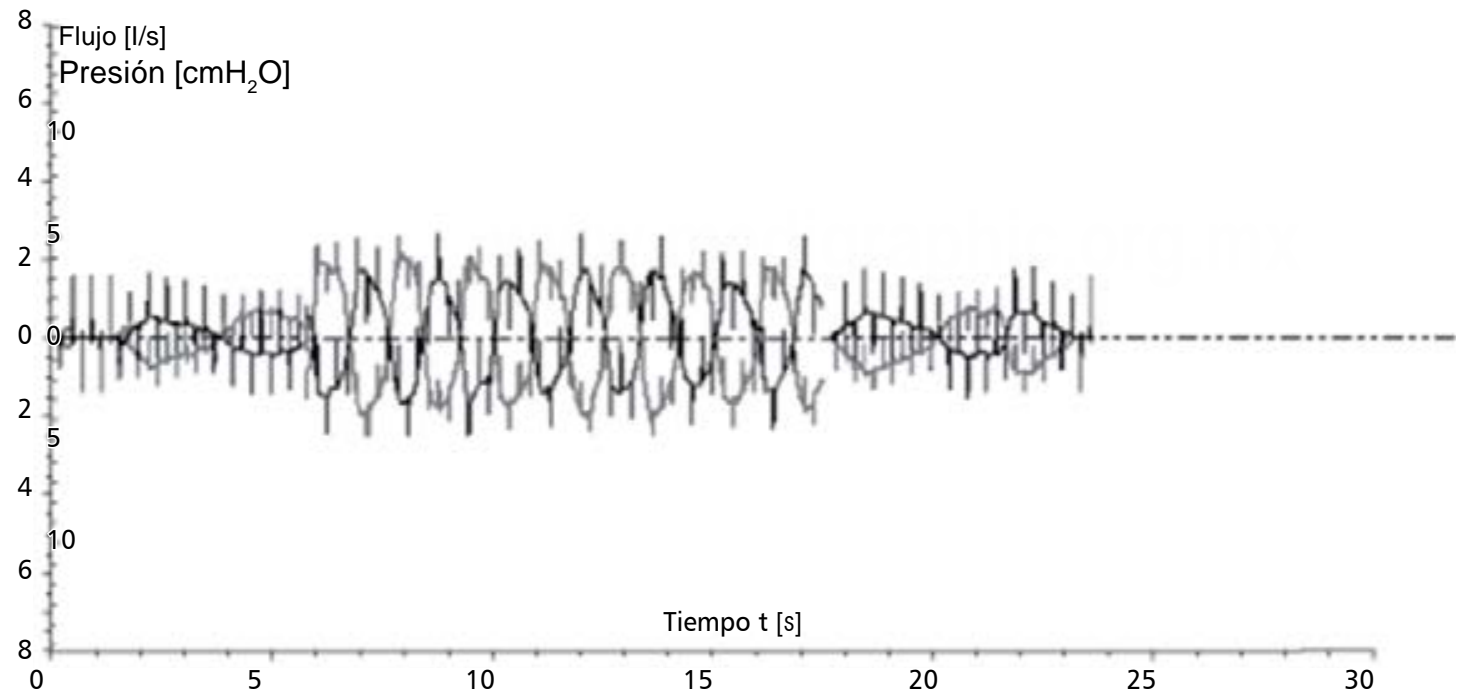

Figura 5:

Artefacto: respiración agitada. 


\begin{tabular}{|l|l|l|}
\hline \multicolumn{3}{|c|}{$\begin{array}{c}\text { NOMBRE DE LA INSTITUCIÓN } \\
\text { «Informe de Oscilometría de Impulso» }\end{array}$} \\
\hline Nombre del paciente: Fernanda González Ruiz & Edad: 7 años & Fecha: 13/09/2012 \\
\hline Fecha de nacimiento: 08/08/2005 & Peso: $30 \mathrm{~kg}$ & \\
\hline Sexo: Femenino & Talla: $134 \mathrm{~cm}$ & \\
\hline
\end{tabular}

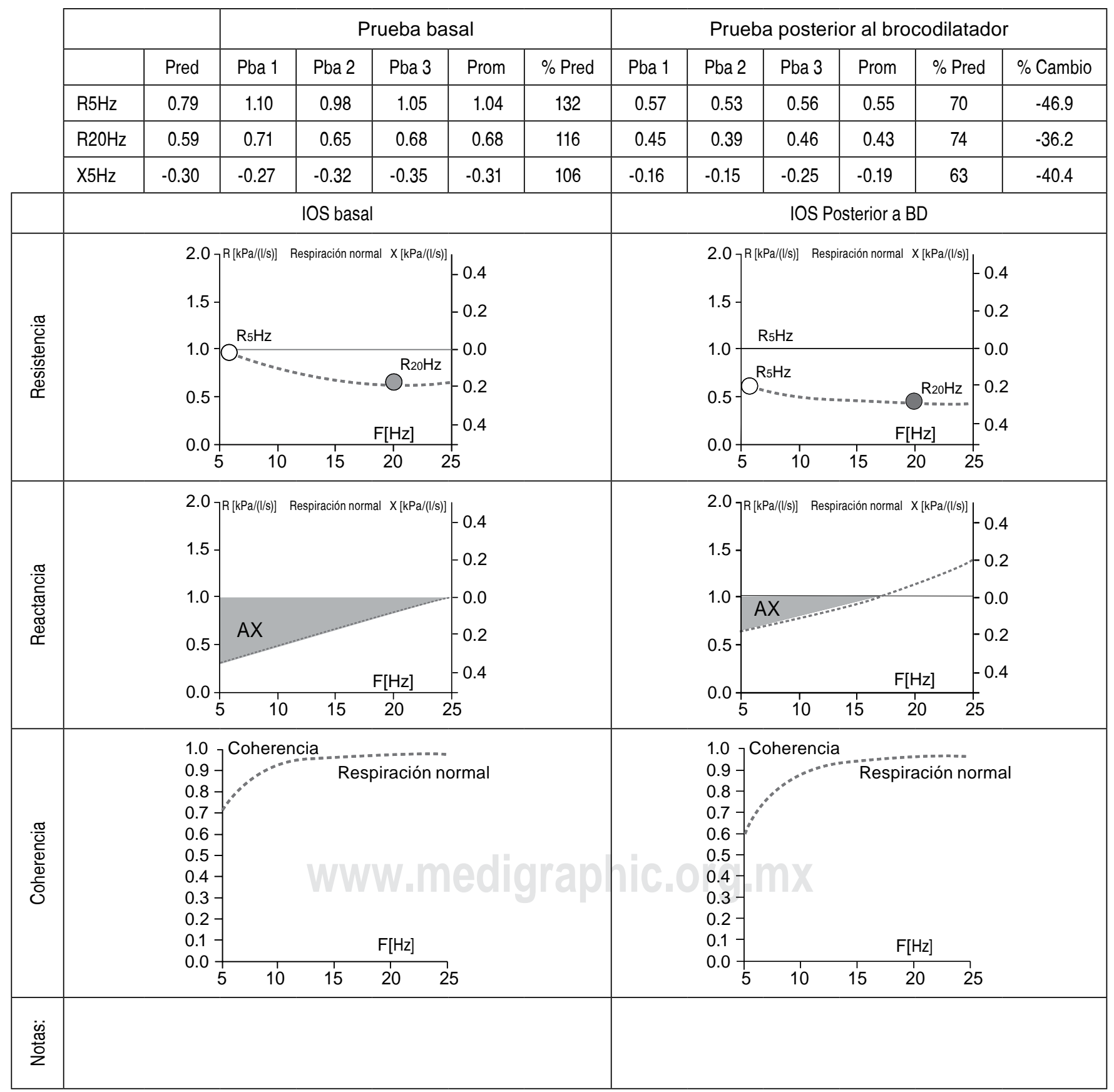

Figura 6: Ejemplo de un informe de oscilometría de impulso. Se muestra un paciente con aumento de las resistencias a expensas de $\mathrm{R} 5 \mathrm{~Hz}$ y con disminución de éstas después de la administración de salbutamol. Existe disminución del área de reactancia (AX). 


\begin{tabular}{|c|c|c|}
\hline & Resistencia (R) & Reactancia (X) \\
\hline $\begin{array}{c}\text { A } \\
\text { Normal }\end{array}$ & 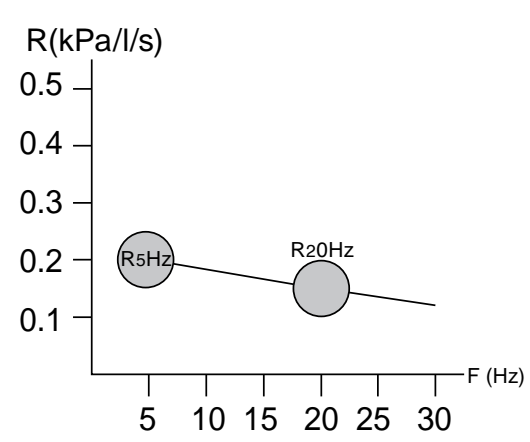 & 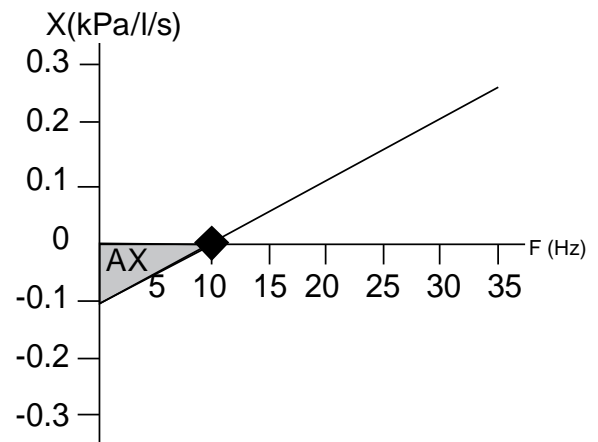 \\
\hline B Obstrucción distal & 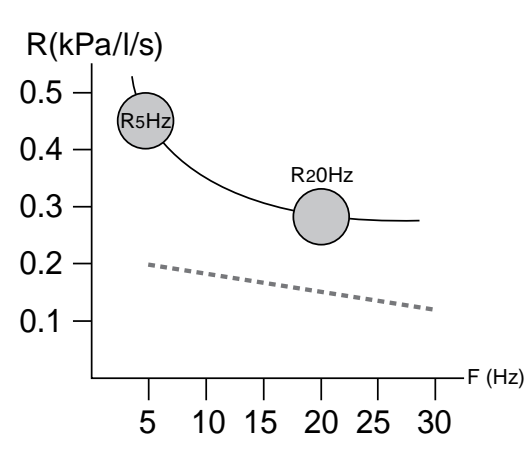 & 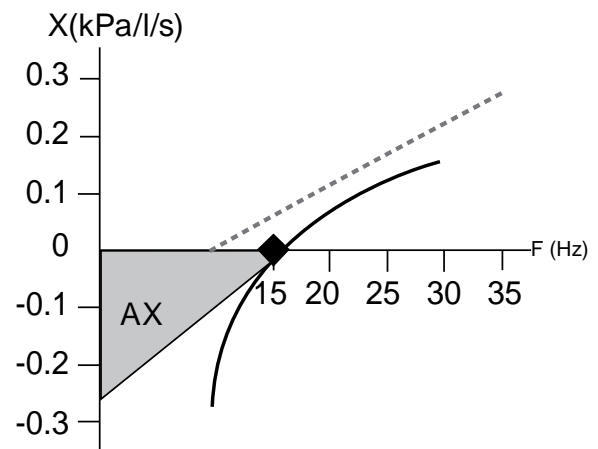 \\
\hline $\begin{array}{c}\text { C } \\
\text { Obstrucción proximal }\end{array}$ & 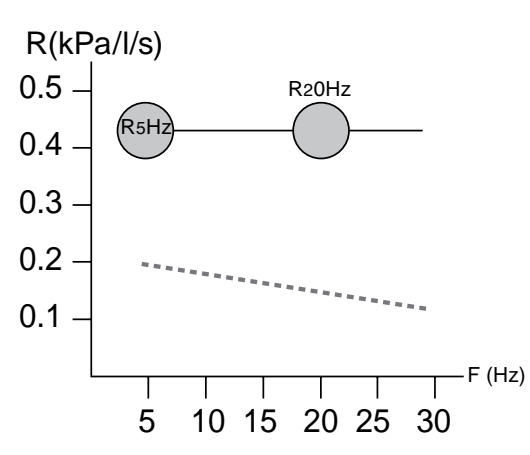 & $\begin{array}{lllllllll}\mathrm{X}(\mathrm{kPa} / \mathrm{l} / \mathrm{s}) & \\
\end{array}$ \\
\hline $\begin{array}{c}\text { D } \\
\text { Sugiere restricción }\end{array}$ & 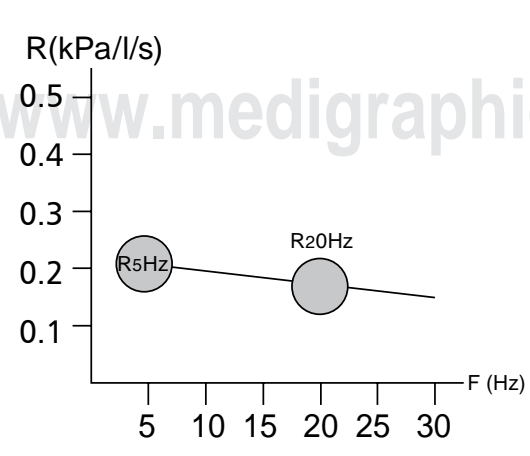 & 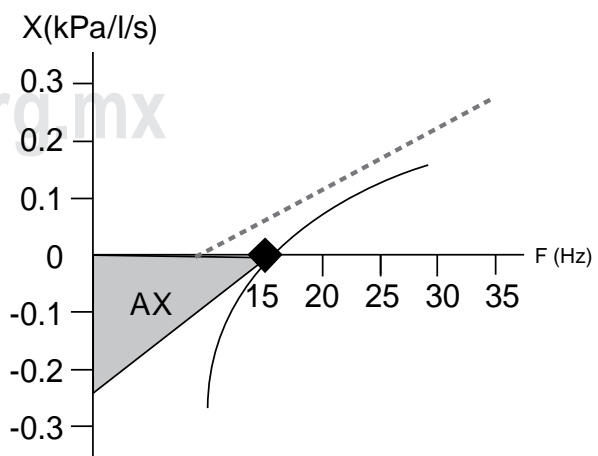 \\
\hline
\end{tabular}

Figura 7: Patrones funcionales que pueden obtenerse mediante la IOS. 


\section{INFORME DE LA IOS}

a. El informe de la oscilometría contendrá todos los valores de $\mathrm{R}$ y $\mathrm{X}$ calculados a los diferentes $\mathrm{Hz}$ antes y después del broncodilatador o, en su caso, todos los valores basales y posteriores a prueba de reto bronquial, especificando las unidades de medición (ej., $\mathrm{kPa} / \mathrm{L} / \mathrm{s}$ o en $\mathrm{CmH}_{2} \mathrm{O} / \mathrm{L} / \mathrm{s}$ ) (Figura 6).

b. Los resultados indispensables son $\mathrm{R} 5 \mathrm{~Hz}, \mathrm{R} 20 \mathrm{~Hz}$, R5-R20 X $5 \mathrm{~Hz}$, Fres y AX.

c. El resultado final es la media de las tres mediciones que cumplieron criterios de aceptabilidad y repetibilidad, tanto para resistencia como para $\mathrm{X}$.

d. Además, se informará la Coeh de las mediciones a
5 y $10 \mathrm{~Hz}$, cuyo valor debe ser de 0.6 y 0.9 , respectivamente.

e. En el informe debe aparecer el predicho seleccionado y el cálculo del porcentaje del predicho. En la Tabla 1 se muestran algunas de las ecuaciones de referencias publicadas hasta la fecha. ${ }^{17-23}$

\section{INTERPRETACIÓN DE LA PRUEBA}

Una vez que se cuenta con una prueba aceptable y repetible, se toman los valores promedios para la interpretación. En la Figura 7 se observan los patrones que pueden obtenerse en la IOS; y en la Figura 8 se presenta una propuesta del algoritmo de interpretación.

Tabla 1. Ecuaciones de referencia de la resistencia y reactancia en adultos y niños.

\begin{tabular}{|c|c|c|c|c|}
\hline Autor & $\begin{array}{l}\text { Sujetos } \\
\text { incluidos }\end{array}$ & Edad & Ecuación & DER EE \\
\hline \multirow{2}{*}{ Newbury $W^{17}$} & \multirow{2}{*}{100} & \multirow{2}{*}{$25-74$} & $\begin{array}{l}\text { Mujeres: } \\
\text { R5 }=0.768+0.00064^{\star} E-0.00276^{\star} T(C L S N=+0.218) \\
\text { R20 }=0.4821+0.00034^{\star} E-0.00125^{\star} T(C L S N=+0.154) \\
X 5=-0.4689-0.00092^{\star} E+0.00245^{\star} T(C L S N=+0.092)\end{array}$ & $\begin{array}{l}\text { Mujeres: } \\
\mathrm{EE}=0.095 \\
\mathrm{EE}=0.067 \\
\mathrm{EE}=0.04\end{array}$ \\
\hline & & & $\begin{array}{l}\text { Hombres: } \\
\text { R5 }=1.1672-0.0017^{\star} E+0.0043^{\star} \mathrm{P}-0.007^{\star} \mathrm{T}(\mathrm{CLSN}=+0.161) \\
\text { R20 }=0.9216-0.0013^{\star} \mathrm{E}+0.0027^{\star} \mathrm{P}-0.0049^{\star} \mathrm{T}(\mathrm{CLSN}=+131) \\
\mathrm{X} 5=-0.3593+0.00013^{\star} \mathrm{E}+0.0016^{*} \mathrm{~T}(\mathrm{CLSN}=+0.055)\end{array}$ & $\begin{array}{l}\text { Hombre: } \\
\mathrm{EE}=0.375 \\
\mathrm{EE}=0.315 \\
\mathrm{EE} 0=.024\end{array}$ \\
\hline Shiota $\mathrm{S}^{18}$ & 166 & $20-83$ & $\begin{array}{l}\text { R5 }=-3.841167^{\star} \log T+8.671580 \\
R 20=-2.546561^{*} \log T+5.841867 \\
X 5=-0.000097^{*} E+1.018597^{*} \log T-2.343672\end{array}$ & $\begin{array}{l}\mathrm{DER}=0.06 \\
\mathrm{DER}=0.05 \\
\mathrm{DER}=0.03\end{array}$ \\
\hline Dencker $\mathrm{M}^{19}$ & 360 & 2.1-11.1 & $\begin{array}{l}\text { R5 }=0.266+0.759^{*} T^{-3}+0.004^{*} P \\
R 20=0.259+0.630^{\star} T^{-3} \\
X 5=-0.123-0.225^{*} T^{-3}\end{array}$ & $\begin{array}{l}\mathrm{DER}=0.188 \\
\mathrm{DER}=0.159 \\
\mathrm{DER}=0.090\end{array}$ \\
\hline Frei $J^{20}$ & 222 & 3-10 & $\begin{array}{l}\text { R5 }=2.11679-0.0099^{*} T \\
\text { R20 }=1.45644-0.0065^{\star} T \\
X 5=-1.1974+0.00673^{\star} T\end{array}$ & $\begin{array}{l}\mathrm{EE}=0.1494 \\
\mathrm{EE}=0.1263 \\
\mathrm{EE}=0.1684\end{array}$ \\
\hline Nowowiejska $B^{21}$ & 626 & $3.1-18.9$ & $\begin{array}{l}R 5=e^{1.818-0.0169 \cdot T} \\
R 20=e^{1.217-0.0148 \cdot T} \\
X 5=e=0.0037 \cdot T-0.728\end{array}$ & $\begin{array}{l}\mathrm{DER}=0.2185 \\
\mathrm{DER}=0.2196 \\
\mathrm{DER}=0.0545\end{array}$ \\
\hline Lee JY²2 & 161 & $3.5-7.17$ & $\begin{array}{l}R 5=2.242-0.008^{*} T-0.005^{\star} E \\
R 20=1.518-0.007^{\star} T \\
X 5=-9.21+0.005^{*} T\end{array}$ & $\begin{array}{l}\mathrm{DER}=0.168 \\
\mathrm{DER}=0.124 \\
\mathrm{DER}=0.113\end{array}$ \\
\hline \multirow{2}{*}{ Amra $B^{23}$} & \multirow{2}{*}{509} & \multirow{2}{*}{ 6-19 } & $\begin{array}{l}\text { Niños: } \\
\text { R5 }=-6.19^{*} 10^{-7 *} E^{\star} 3.820-6.78^{*} 10^{-5 *} T^{*} 1.651+0.691 \\
\text { R25 }=-6.71^{*} 10^{-7 *} E^{\star} 3.75-8.7^{*} 10^{-10^{*}} T^{*} 3.57+0.362 \\
X 5=6.95^{*} 10^{-23} E^{\star} 16.226+0.004^{*} T^{\star} 0.846-0.430\end{array}$ & $\begin{array}{l}\mathrm{EE}=0.001 \\
\mathrm{EE}=0.0000 \\
\mathrm{EE}=0.061\end{array}$ \\
\hline & & & $\begin{array}{l}\text { Niñas: } \\
\text { R5 }=-1.35^{\star} 10^{-5 *} E^{\star} 2.823-0.001^{\star} T^{\star} 1.022+0.547 \\
\text { R25 }=0.14^{\star} E^{\star} 0.254-8.66^{*} 10^{-6 *} T^{\star} 1.759-0.341 \\
X 5=1.78^{\star} 10^{-7 *} E^{\star} 4.15+0.002^{*} T^{\star} 1.08-0.539\end{array}$ & $\begin{array}{l}\mathrm{EE}=0.049 \\
\mathrm{EE}=0.00 \\
\mathrm{EE}=0.022\end{array}$ \\
\hline
\end{tabular}

R5 = Resistencias a $5 \mathrm{~Hz}$; R20 = Resistencias a $20 \mathrm{~Hz}$; X5 = Reactancia a $5 \mathrm{~Hz}$; DER = Desviación estándar de los residuales;

$\mathrm{EE}=$ Error estándar; $\mathrm{E}=\mathrm{Edad} ; \mathrm{T}=$ Talla $(\mathrm{cm}) ; \mathrm{P}=\mathrm{Peso}(\mathrm{kg}) ; \mathrm{CLSN}=$ Constante para predecir el límite superior de la normalidad; LogT = Logaritmo de la talla. 


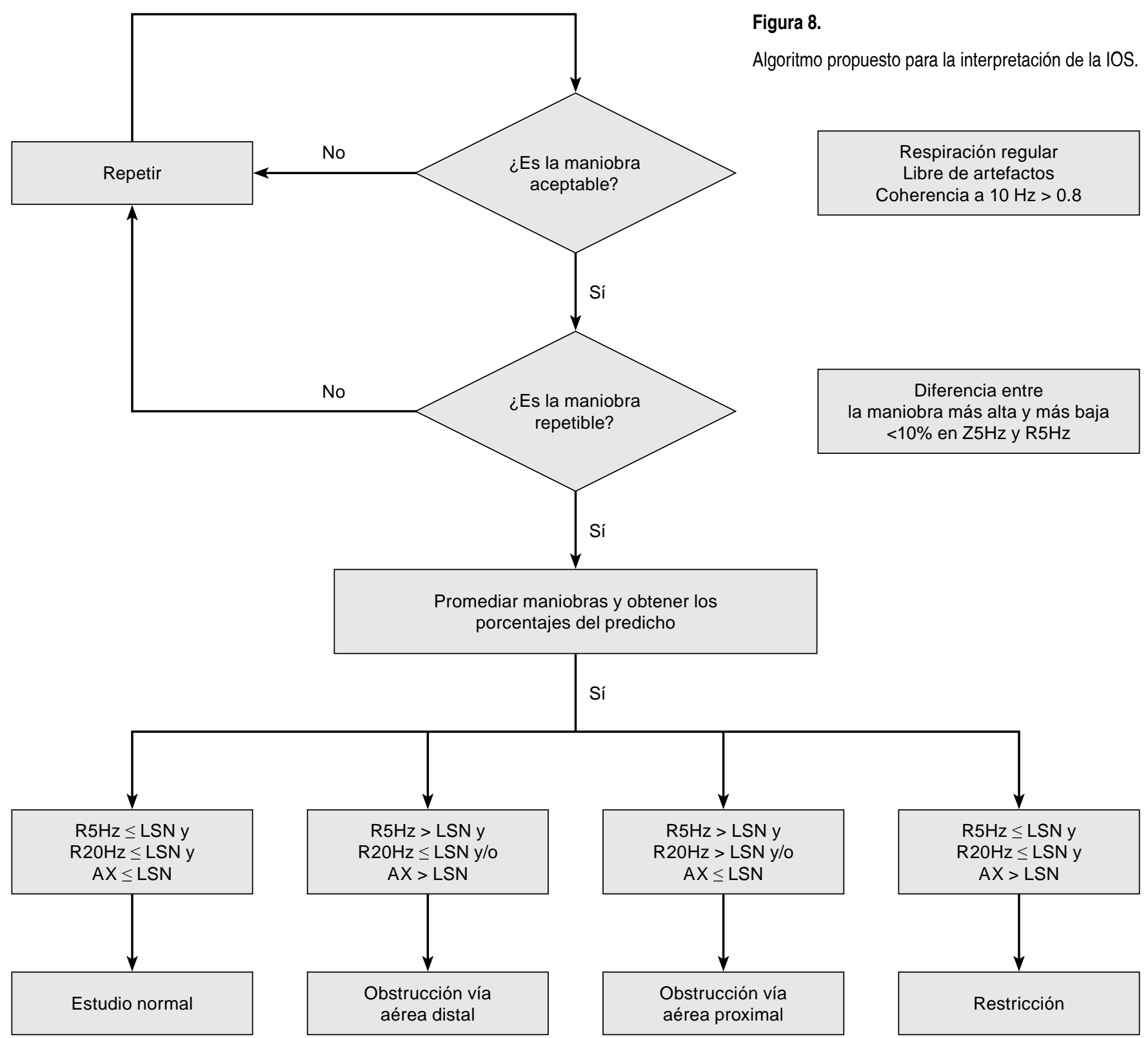

Se considera una IOS normal cuando la R $5 \mathrm{~Hz}$, la R 20 $\mathrm{Hz}$ y el AX se encuentran por debajo del límite superior de la normalidad (LSN) o en \pm 1.64 valores $Z$ del predicho. También se han considerado valores normales a aquellos que no excedan el $150 \%$ del valor predictivo, lo que sería equivalente a una disminución del $20 \%$ del volumen espiratorio forzado durante el primer segundo $\left(\mathrm{VEF}_{1}\right)$.

Si el valor de $\mathrm{R} 5 \mathrm{~Hz}$ se encuentra por arriba del LSN, o > de 1.64 valores $Z$ del predicho, con $R 20 \mathrm{~Hz}$ y AX normal, el resultado habla de obstrucción distal y se clasifica de acuerdo al valor $Z$, cuando éste se encuentra entre 1.64 y 2 desviaciones estándar (DE) la obstrucción es leve, si es $\geq 2 \mathrm{DE}$ es moderada y si es $\geq 4 \mathrm{DE}$ se considera grave.
Este patrón es frecuente en pacientes con asma donde la $\mathrm{R}$ aumenta a bajas frecuencias de oscilación y disminuye en altas frecuencias de oscilación; es decir, el aumento de las $\mathrm{R}$ es a expensas de $\mathrm{R} 5 \mathrm{~Hz}$.

Cuando la R $5 \mathrm{~Hz}$ y la R $20 \mathrm{~Hz}$ se encuentran por arriba del LSN y el AX es normal, el patrón de obstrucción se considera central o proximal ya que el aumento de $\mathrm{R}$ es independiente de la frecuencia de oscilación. Se ha sugerido que cuando las $\mathrm{R}$ a $5 \mathrm{~Hz}$ y $20 \mathrm{~Hz}$ se encuentran normales con un $A X \geq L S N$, sugiere un patrón restrictivo.

La interpretación con base en la $X$ aún está en fase de estudio dada la gran variabilidad que se encuentra en los resultados. 
Cuando se aplica broncodilatador, la IOS se considera positiva cuando existe una disminución en las resistencias a $5 \mathrm{~Hz}$ de más del $20 \%$. La prueba también ha sido estudiada en reto bronquial con ejercicio y se considera positiva cuando existe un aumento del $35 \%$ en las resistencias a $5 \mathrm{~Hz}$, lo cual comúnmente ocurre a los 2 o 5 minutos; o un aumento de $0.035 \mathrm{kPa} / \mathrm{L} / \mathrm{s}$ a los 10 minutos en las resistencias a $5 \mathrm{~Hz}$.

La prueba de reto bronquial con metacolina ha sido menos estudiada y se ha propuesto utilizar la X $5 \mathrm{~Hz}$; pero como hemos mencionado, la medición de la $\mathrm{X}$ ha presentado gran variabilidad. ${ }^{2}$ La prueba de reto bronquial con manitol se considera positiva cuando existe un aumento de más de $25 \%$ en las $\mathrm{R}$ a $5 \mathrm{~Hz} .^{13}$

\section{CONCLUSIONES}

La IOS es una prueba que ha sido estandarizada para la evaluación no solo de pacientes poco cooperadores, sino que, además, es una prueba complementaria a otros estudios. Es importante realizarla e interpretarla de forma estandarizada y con un adecuado control de calidad.

\section{REFERENCIAS}

1. Vargas-Domínguez $\mathrm{C}$, Gochicoa-Rangel L, Velázquez-Uncal M, et al. Pruebas de función respiratoria, ¿cuál y a quién? Neumol Cir Torax 2011;70(2):101-117.

2. Beydon N, Davis SD, Lombardi E, et al.; American Thoracic Society/ European Respiratory Society Working Group on Infant and Young Children Pulmonary Function Testing. An official American Thoracic Society/European Respiratory Society statement: pulmonary function testing in preschool children. Am J Respir Crit Care Med 2007;175(12):1304-1345.

3. Dubois AB, Brody AW, Lewis DH, Burgess BF Jr. Oscillation mechanics of lungs and chest in man. J Appl Physiol 1956;8(6):587-594.

4. Al-Mutairi SS, Sharma PN, Al-Alawi A, Al-Deen JS. Impulse oscillometry: an alternative modality to the conventional pulmonary function test to categorise obstructive pulmonary disorders. Clin Exp Med 2007;7(2):56-64.

5. Smith HJ, Reinhold P, Goldman MD. Forced oscillation technique and impulse oscillometry. Eur Respir Mon 2005;31:72-105.

6. Pride NB. Forced oscillation techniques for measuring mechanical properties of the respiratory system. Thorax 1992;47(4):317-320.

7. Larsen GL, Morgan W, Heldt GP, et al.; Childhood Asthma Research and Education Network of the National Heart, Lung, and Blood Institute. Impulse oscillometry versus spirometry in a long-term study of controller therapy for pediatric asthma. J Allergy Clin Immunol 2009;123(4):861-867.
8. Voter KZ, McBride JT. Diagnostic tests of lung function. Pediatr Rev 1996;17(2):53-63.

9. Edwards CA, Osman LM, Godden DJ, Douglas JG. Wheezy bronchitis in childhood: a distinct clinical entity with lifelong significance? Chest 2003; 124(1):18-24.

10. Ortiz G, Menendez R. The effects of inhaled albuterol and salmeterol in 2- to 5-year-old asthmatic children as measured by impulse oscillometry. J Asthma 2002;39(6):531-536.

11. Vogel J, Smidt U, editors. Impulse oscillometry: analysis of lung mechanics in general practice and the clinical, epidemiological and experimental research. Frankfurt: Verlagsgruppe; 1994.

12. Kim HY, Shin YH, Jung da W, Jee HM, Park HW, Han MY. Resistance and reactance in oscillation lung function reflect basal lung function and bronchial hyperresponsiveness respectively. Respirology 2009;14(7):1035-1041.

13. Malmberg LP1, Mäkelä MJ, Mattila PS, Hammarén-Malmi S, Pelkonen AS. Exercise-induced changes in respiratory impedance in young wheezy children and nonatopic controls. Pediatr Pulmonol 2008;43(6):538-544.

14. Mauer MP, Cummings KR. Impulse oscillometry and respiratory symptoms in World Trade Center responders, 6 years post-9/11. Lung 2010;188(2):107-113.

15. Horsman TA, Duke RK, Davenport PW. Airway response to mannitol challenge in asthmatic children using impulse oscillometry. J Asthma 2009;46(6):600-603.

16. Jee HM, Kwak JH, Jung da W, Han MY. Useful parameters of bronchial hyperresponsiveness measured with an impulse oscillation technique in preschool children. J Asthma 2010;47(3):227-232.

17. Newbury W, Crockett A, Newbury J. A pilot study to evaluate Australian predictive equations for the impulse oscillometry system. Respirology 2008;13(7):1070-1075.

18. Shiota S, Katoh M, Fujii M, Aoki S, Matsuoka R, Fukuchi Y. Predictive equations and the reliability of the impulse oscillatory system in Japanese adult subjects. Respirology 2005;10(3):310-315.

19. Dencker M, Malmberg LP, Valind S, et al. Reference values for respiratory system impedance by using impulse oscillometry in children aged 2-11 years. Clin Physiol Funct Imaging 2006;26(4):247-250.

20. Frei J, Jutla J, Kramer G, Hatzakis GE, Ducharme FM, Davis GM. Impulse oscillometry: reference values in children 100 to $150 \mathrm{~cm}$ in height and 3 to 10 years of age. Chest 2005;128(3):1266-1273.

21. Nowowiejska B, Tomalak W, Radli ski J, Siergiejko G, Latawiec W, Kaczmarski M. Transient reference values for impulse oscillometry for children aged 3-18 tears. Pediatr Pulmonol 2008;43(12):1193-1197.

22. Lee JY, Seo JH, Kim HY, et al. Reference values of impulse oscillometry and its utility in the diagnosis of asthma in young Korean children. $J$ Asthma 2012;49(8):811-816.

23. Amra B, Soltaninejad F, Golshan M. Respiratory resistance by impulse oscillometry in healthy Iranian children aged 5-19 years. Iran J Allergy Asthma Immunol 2008;7(1):25-29.

Los autores declaran no tener conflicto de intereses. 